\title{
Allgemeines Schuldrecht
}

\author{
Inhaltsübersicht
}

A. Ausgangslage in der ersten Hälfte des 20. Jahrhunderts
B. Überblick über die Entwicklung der Schuldrechtsliteratur des Verlages
I. Ausgangspunkt des Verlages im Allgemeinen Schuldrecht
II. Ausbau des Verlagsprogramms im Allgemeinen Schuldrecht
III. Eroberung der führenden Stellung
IV. Reaktion auf die Umgestaltung des Allgemeinen Schuldrechts
V. Aktuelle Funktionen von Kommentaren und Lehrbüchern
C. Weiterentwicklung des Allgemeinen Schuldrechts
I. Einfluß der Grundrechte
II. Entwicklungen im Schadensersatzrecht
1. Ausdehnung des immateriellen Schadens
2. Kommerzialisierung beim materiellen Schaden?
3. Vordringen des normativen Schadensbegriffs
4. Schutzzweck der Norm
5. Vertrag mit Schutzwirkung für Dritte
6. Gründe für die Entwicklung im Schadensersatzrecht
III. § 242 BGB als Grundlage für die Fortentwicklung des Schuldrechts
IV. Entwicklungen im Recht der Forderungsabtretung
V. Schuldrechtsmodernisierung
D. Ein Blick in die Zukunft
I. Zukunft des Allgemeinen Schuldrechts
II. Buchdruck und elektronische Medien
III. Zukunft des Verlages

Das Allgemeine Schuldrecht gehört zum zentralen Lehrstoff des Zivilrechts schon in den ersten Semestern. Das Schuldverhältnis als die Sonderverbindung mit Rechten und Pflichten zwischen zwei Personen betrifft das Grundverständnis der rechtlich geregelten Lebensverhältnisse in Gesellschaft und Wirtschaft. Die Erfüllung des Schuldverhältnisses, Art und Weise der ordnungsgemäßen Leistungserbringung sind ebenso Gegenstand des Allgemeinen Schuldrechts wie die Pflichtverletzungen im Zusammenhang mit Leistungsstörungen und die daran geknüpften Rechtsfolgen von Rücktritt und Schadensersatz. Forderungsabtretung, Aufrechnung und Gesamtschuld sind weitere Regelungsbereiche mit täglicher Relevanz für die Praxis. Die Bedeutung des Allgemeinen Schuldrechts wird noch dadurch unterstrichen, daß es sowohl für vertragliche wie für gesetzliche Schuldverhältnisse zur Anwendung kommt. Dies alles ist mit dem für einen allgemeinen Teil typischen Abstraktionsgrad verbunden, der dem Verständnis und der Anschaulichkeit für den jungen Juristen nicht gerade förderlich ist. 


\section{A. Ausgangslage in der ersten Hälfte des 20. Jahrhunderts}

Lehrbücher mit erläuternden Beispielen und Fallsammlungen mit Fallösungen wären angesichts des hohen Abstraktionsgrads im Allgemeinen Schuldrecht für den jungen Juristen hilfreiche Lernmittel gewesen. Sie waren aber nicht im heutigen Umfang und der heutigen Auswahl vorhanden. Dies entsprach nicht dem allgemeinen Verständnis in der ersten Hälfte des 20. Jahrhunderts. Auch der Verlag C. H. Beck hatte den Lehrbuchmarkt zu dieser Zeit noch nicht richtig erschlossen. Im Allgemeinen Schuldrecht weist der Verlagskatalog am Anfang der 30er Jahre gerade einmal das Lehrbuch des Allgemeinen Schuldrechts von Geh. Justizrat Prof. Dr. Hugo Kreß aus dem Jahre 1928 aus. Um diese Zeit entstand auch die Reihe „Prüfe Dein Wissen, Rechtsfälle und Fragen mit Antworten“ verfaßt von Dr. Heinrich Schönfelder. Dazu gehörte ein Band Schuldrecht, Allgemeine Lehren. Dabei handelte es sich zumeist um kleine Fälle mit Fragen und Lösungen, die nicht mit den umfangreichen Fall- und Klausurlösungen späterer Zeit vergleichbar waren.

Das große Lehrbuch zum Recht der Schuldverhältnisse von Enneccerus, das später von Heinrich Lehmann fortgeführt wurde, ist im Verlag Mohr (Siebeck) erschienen ebenso wie das an der Methode der Interessenjurisprudenz orientierte Lehrbuch zum Schuldrecht von Philipp Heck. Von anderen Verlagen stammen auch die Lehrbücher zum Deutschen bürgerlichen Recht von Konrad Cosack und von Heinrich Dernburg. Auf diese Lehrbüchern konnten und mußten die Studenten noch lange Zeit bis in die fünfziger Jahre des 20. Jahrhunderts zurückgreifen. Im Verlag Beck war nach dem zweiten Weltkrieg noch das Kurzlehrbuch zum Schuldrecht von Erich Molitor erschienen. Das zweibändige Werk behandelte in Band 1 das Allgemeine Schuldrecht, konnte sich gegen die großen Lehrbücher aber nicht so recht durchsetzen.

Die verhaltene Entwicklung der Lehrbücher zum Schuldrecht mit eher langfristiger Auflagenfolge hat mehrere Ursachen. Ein Grund mag wohl die mäßige Entwicklung der Studentenzahlen in der ersten Hälfte des 20. Jahrhunderts gewesen sein. Noch Ende der 50er Jahre betrug die Zahl der Erstsemester in der juristischen Fakultät der Universität Tübingen etwa 80 Studenten. 1972 studierten in Frankfurt dagegen insgesamt ca. 1600 Studenten Jura. In den Jahren 1990 bis 2000 betrug die Zahl der Anfänger im Fachbereich Rechtswissenschaft im Wintersemester jeweils ca. 500 Studenten, im Sommersemester ca. 250 Studenten. Die Gesamtzahl der Juristen betrug 2004 in Frankfurt etwa 4000 Studenten. Diese vermehrten Studentenzahlen erklären die erhöhte Nachfrage nach Lehrbüchern in der zweiten Hälfte des 20. Jahrhunderts.

Ein weiterer Grund lag wohl in einer vergleichsweise konstanten Entwicklung des allgemeinen Schuldrechts. Konstant war diese Entwicklung vor allem, weil die Vorschriften und Rechtsfiguren des Allgemeinen Schuldrechts in ihrer abstrakten und generellen Ausformung so flexibel waren, daß sie die gesellschaftlichen und wirtschaftlichen Veränderungen ohne wesentliche Auslegungsprobleme in sich aufnehmen konnten. Auch scheint es, daß die Juristen früher im abstrakten Den- 
ken besser geübt waren und nicht hinter jeder Fallvariante einen neuen Rechtssatz $\mathrm{zu}$ entdecken versuchten. Zwar wurden in den ersten Jahren und Jahrzehnten nach dem Inkrafttreten des BGB mit der culpa in contrahendo und der positiven Vertragsverletzung wichtige Rechtsfiguren im wesentlichen von der Wissenschaft entwickelt. Ebenso hatten schwere wirtschaftliche Erschütterungen vor und nach dem ersten Weltkrieg Anlaß zur Fortentwicklung der Lehre vom Wegfall der Geschäftsgrundlage gegeben. Nachdem diese Rechtsfiguren in die Lehrbücher aufgenommen und theoretisch verarbeitet worden waren, gaben sie in ihrer abstrakten Ausgestaltung aber zu keinen weiteren Entwicklungen Anlaß. Sie beschäftigten zwar immer wieder die Praxis und die Rechtsprechung, weil sie zu Schwierigkeiten in der konkreten Anwendung führten. Ihren theoretischen Geltungsanspruch und ihre lehrbuchhafte Ausgestaltung berührte dies jedoch nicht entscheidend.

Ein weiterer Grund für die verhaltene Entwicklung der Lehrbücher mögen auch die eher unruhigen Verhältnisse in der ersten Hälfte des 20. Jahrhunderts mit zwei Weltkriegen, Inflation, Wirtschaftskrise und der Zeit des Nationalsozialismus gewesen sein. Hinzu kamen die Studentenzahlen, die verglichen mit der heutigen Zeit eher gering waren. Dagegen gab es aber auch einige Kommentare zum BGB, wie die Großkommentare von Planck und Staudinger sowie den Kommentar der Reichsgerichtsräte oder den Kommentar von Oertmann, die den Juristen in Ausbildung und Beruf wertvolle Hilfe boten. Auch Kurzkommentare zum BGB, unter denen auch der Kommentar von Palandt seinen Anfang nahm, standen neben sonstigen kleineren Hilfsmitteln zur Verfügung. Die literarische Versorgung war danach in der ersten Hälfte des 20. Jahrhunderts auf dem Gebiet des Allgemeinen Schuldrechts durchaus vielfältig und beachtlich. In den Jahren des zweiten Weltkriegs konnte sich die Rechtswissenschaft allerdings nicht wesentlich weiterentwickeln.

\section{B. Überblick über die Entwicklung der Schuldrechtsliteratur des Verlages}

Als der BGH im Jahre 1950 seine Tätigkeit aufnahm, erlebte die Zivilrechtwissenschaft eine neue Blüte. Bis zur Modernisierung des Schuldrechts im Jahr 2002 blieb der Gesetzestext im Allgemeinen Teil des Schuldrechts nahezu unverändert. Die Rechtsentwicklung wurde hauptsächlich von der Rechtsprechung und der Wissenschaft vorangetrieben. Dabei griff sowohl die Rechtsprechung Gedanken aus der Rechtswissenschaft auf und verhalf ihnen zum Durchbruch und zu praktischer Geltung. Umgekehrt trug die Rechtsprechung mit neuen Überlegungen, die von der Rechtswissenschaft aufgegriffen, systematisch eingeordnet und weiter ausgebaut wurden, zur Fortentwicklung des Rechts bei.

\section{Ausgangspunkt des Verlages im Allgemeinen Schuldrecht}

Der Verlag C.H. Beck hat zu dieser Entwicklung mit seinen Lehrbüchern und Kommentaren im Bereich des Allgemeinen Schuldrechts zu Beginn nur mit wenigen Werken beigetragen. Der Katalog aus dem Jahre 1949, der damals noch unter 
der Firma Biederstein Verlag, hervorgegangen aus der C.H. Beck'schen Verlagsbuchhandlung, erschien, weist für das Allgemeine Schuldrecht unter den juristischen Kurzlehrbüchern gerade einmal das Schuldrecht, Band I, Allgemeiner Teil in 2. Auflage von Erich Molitor aus. Der Jurist in der Praxis und im Studium konnte allerdings schon damals auf den „Palandt“ in 7. Auflage als Hilfsmittel zurückgreifen.

Der nächste Katalog im Jahr 1950 firmierte bereits unter dem Namen Verlag C.H. Beck. Für das Allgemeine Schuldrecht enthielt er jedoch keine Neuerscheinungen. Lediglich der Palandt erschien in jährlicher Neuauflage. In der Reihe Prüfe dein Wissen wurde 1951 die 3. Bearbeitung des Schuldrechts, Allgemeine Lehren, aufgelegt. Im Jahre 1953 erschien die erste Auflage des großen Lehrbuchs von Larenz zum Schuldrecht, Allgemeiner Teil. Für die Studenten, aber auch für die Praxis war dies eine wesentliche Bereicherung, da mit diesem Lehrbuch eine leicht lesbare umfassende wissenschaftliche Darstellung des aktuellen Schuldrechts vorlag. Im selben Jahr kam das Allgemeine Schuldrecht von Molitor in 3. Auflage. Obwohl das Verlagsprogramm in den folgenden Jahren rasch und stetig anwuchs, gab es im Allgemeinen Schuldrecht keine Neuerscheinungen. Jedoch erschienen die bereits vorhandenen Lehrbücher von Molitor und Larenz mehrfach in neuer Auflage.

\section{Ausbau des Verlagsprogramms im Allgemeinen Schuldrecht}

Erst im Jahre 1969 brachte der Verlag zum ersten Mal die Reihe Grundrisse des Rechts heraus, worin zuerst das Werk von Brox mit dem Titel „Allgemeines Schuldrecht" erschien. Die neue Reihe sollte den wesentlichen Lehrstoff zum Einstieg für den Studenten darstellen, gleichzeitig aber auch dem fortgeschrittenen Studenten die Wiederholung des Stoffes ermöglichen. Die neue Reihe sprach die Bedürfnisse der Studenten nach einer raschen Orientierung über die wichtigen Grundlagen der jeweiligen Rechtsgebiete an. Sie war auch eine Antwort auf das wachsende Konkurrenzangebot der Repetitorien. Das Verlagsangebot reagierte auf die veränderten Bedürfnisse der Studenten auch mit der Herausgabe von fallorientierten Darstellungen, die zum Teil an die Anfangssemester gerichtet waren und die den Stoff etwas ausführlicher darstellten als die vor allem zur Wiederholung und Vertiefung angebotene Reihe „Prüfe dein Wissen“. Im Schuldrecht erschien die fallorientierte Darstellung für Anfangssemester mit dem Titel „Das Schuldverhältnis“ von Löwisch zum ersten Mal 1975 in der JuS-Schriftenreihe. Andere Darstellungen wandten sich mehr an die fortgeschrittenen Studenten und behandelten spezielle Bereiche des Allgemeinen Schuldrechts, wie das 1976 erschienene Werk „Juristischer Studienkurs Leistungsstörungen und Gewährleistung“ von Teichmann sowie das 1978 erschienene Buch zum Recht der Leistungsstörungen von Emmerich. Fälle zum Allgemeinen Schuldrecht behandelte Kornblum in seiner 1978 erschienen Darstellung, die sich an den Anforderungen für die Lösung von Klausuren orientierte.

Das Schuldrecht bot sich als Einstieg in die neuen Reihen, insbesondere auch in die Reihe „Grundrisse des Rechts“ an, weil es den wesentlichen Wissensstoff und 
die maßgeblichen Grundlagen für das Verständnis des Zivilrechts enthielt, woran kein Student vorbeigehen konnte. Außerdem war auch das Lehrbuch von Molitor in der Kurzlehrbuch-Reihe 1965 in letzter Auflage erschienen und stand in keiner aktuellen Bearbeitung mehr zur Verfügung. Erst 1981 konnte in der KurzlehrbuchReihe wieder das Allgemeine Schuldrecht angeboten werden. Vor allem wegen der seit 1965 verstrichenen langen Zeit war eine völlige Neubearbeitung nötig, die eine Fortsetzung des bisherigen Werkes von Molitor ausschloß. Medicus leistete mit gutem Durchblick die immense Arbeit und startete das Werk mit einer neuen ersten Auflage, gefolgt vom Schuldrecht, Besonderer Teil, im Jahre 1983.

Das für Wissenschaft und Lehre sowie in der täglichen Praxis wichtige Allgemeine Schuldrecht wurde vom Verlag nicht nur durch Lehr- und Studienbücher bedient. Vielmehr bietet der Verlag mit seinen Kommentaren zum BGB auch andere wichtige Hilfsmittel an. Nachdem lange Zeit bis zur 37. Auflage allein der BGBKommentar von Palandt in der Reihe Beck'sche Kurz-Kommentare zur Verfügung stand, startete der Verlag im Jahre 1978 mit dem Band 1, Allgemeiner Teil, die Herausgabe des Münchener Kommentars als Großkommentar zum BGB. Die Kommentierung des Allgemeinen Schuldrechts folgte kurz darauf im Jahre 1979 als Band 2 des Münchener Kommentars. Ebenfalls 1979 rundete der Verlag sein Angebot der BGB-Kommentare durch den Kurzkommentar von Jauernig in der „Orangen Reihe“ nach unten ab. Seit 2003 liegt das neueste Kommentarwerk zum BGB von Bamberger/Roth in drei Bänden geschlossen vor und bietet damit eine weitere Kommentierung zum Allgemeinen Schuldrecht. Der neue Kommentar ist zwischen dem Kurzkommentar von Palandt und dem Münchener Kommentar aufgestellt.

\section{Eroberung der führenden Stellung}

Die Lehrbuchreihen und die Kommentare sind bis heute erfolgreiche und geschätzte Hilfsmittel. Dies zeigt allein schon die Zahl der Auflagen. Das große Lehrbuch von Larenz, das gemessen an der Zahl der BGH-Zitate den größten Einfluß auf die Praxis ausübte, ist bis 1987 in 14. Auflage erschienen, das Kurzlehrbuch von Medicus, das in erster Linie als Studienliteratur konzipiert ist, hat in der kurzen Zeit seit 1981 insgesamt 16 Auflagen erlebt. Der ebenfalls als Studienliteratur konzipierte Grundriß von Brox, der ab der 28. Auflage von Walker übernommen wurde, liegt im Jahre 2006 sogar in 33. Auflage vor.

Bei den Kommentaren liegt der „Palandt“ mit 66 Auflagen an der Spitze. Mit ihm hat der Beckverlag den herausragenden Standardkommentar geschaffen, der durch sein jährliches Erscheinen die Möglichkeit zur zuverlässigen Information über die aktuellen Entwicklungen auf dem Gebiet der zivilistischen Gesetzgebung und vor allem der höchstrichterlichen und obergerichtlichen Rechtsprechung gibt. In ihm sind auch, vor allem in den von Heinrichs bearbeiteten Teilen, die wichtigsten wissenschaftlichen Streitfragen dargestellt. Der Kommentar ist deshalb sowohl für die Praxis wie für Wissenschaft und Studium eine unentbehrliche Informationsquelle und ist auf oder zumindest nahe bei dem Arbeitstisch jedes ernsthaften Ziviljuristen, insbesondere des Zivilrichters und Zivilanwalts $\mathrm{zu}$ finden, wird aber auch in den Bibliotheken und Juristischen Seminaren mehrfach angeschafft. 
Der Kurzkommentar von Jauernig dient von seiner Anlage her der Information über die grundlegenden Fragen der Auslegung und Anwendung der Vorschriften des BGB, gibt aber auch zuverlässige Auskunft über Einzelfragen. Der Kommentar hat es bis heute auf 12 Auflagen gebracht.

Für den Münchener Kommentar sind als Großkommentar die Neuauflagen in weniger rascher Folge vorgesehen. Aber auch hier ist die 4. Auflage erschienen und die 5. Auflage im Erscheinen begriffen. Der Kommentar gibt schon aufgrund seines Umfangs in 13 Bänden der 4. Auflage die Möglichkeit zur gründlichen und ausführlichen Darstellung der einzelnen Probleme der jeweiligen Vorschriften des BGB. Dabei nehmen die Autoren regelmäßig auch die Gelegenheit wahr, sich zu Einzelfragen kritisch zu äußern und ihre eigene Meinung zur Geltung zu bringen. Damit ist der Kommentar sowohl für den Wissenschaftler und Studenten als auch für den an einer vertieften Problembehandlung interessierten Praktiker von höchstem Interesse. Aufgrund der geschickten und bewährten Strategie des BeckVerlags ist es auch in kurzer Zeit gelungen, den Kommentar zum auflagenstärksten Großkommentar zu machen.

Selbständige Formularbücher spielen im Bereich des Allgemeinen Schuldrechts naturgemäß wegen der Allgemeinheit und Breite seines Anwendungsbereichs keine Rolle. Auch gibt das Allgemeine Schuldrecht kaum Anlaß, Formulare auszuarbeiten. Soweit sich Probleme stellen, werden sie bei den mit speziellen Sachfragen befaßten Werken angesprochen.

\section{Reaktion auf die Umgestaltung des Allgemeinen Schuldrechts}

Die Schuldrechtsmodernisierung im Jahre 2002, die auch im Allgemeinen Teil wesentliche Veränderungen brachte, hat sich ebenfalls auf das Verlagsprogramm ausgewirkt. Noch im Jahre 2002 sind einige Werke erschienen, die sich dem neuen Schuldrecht widmen. Zu nennen sind Huber/Faust, Schuldrechtsmodernisierung, daneben Haas/Medicus/Rolland/Schäfer/Wendtland, Das neue Schuldrecht, ferner Lorenz/Riehm, Lehrbuch zum neuen Schuldrecht und Ehmann/Sutschet, Modernisiertes Schuldrecht. Solange die Lehrbücher und Kommentare die durch die Schuldrechtsmodernisierung bewirkten Änderungen nicht berücksichtigen konnten, konnte man sich in den neuen Büchern über die Änderungen informieren. Nachdem aber die eingeführten Lehrbücher und Kommentare die neuen Vorschriften verarbeitet haben, bieten sie die nötige Information, und die neuen Werke dürften es schwer haben. Einem längerfristigen Bedürfnis der Studenten, vor allem der Studienanfänger dürfte aber die neue Reihe Juristische Fall-Lösungen entgegenkommen, worin Fritzsche Fälle zum Schuldrecht I und damit zum Schuldrecht, Allgemeiner Teil, bearbeitet hat.

\section{Aktuelle Funktionen von Kommentaren und Lehrbüchern}

Zusammenfassend kann damit festgehalten werden: Der Erfolg der Lehrbücher und Kommentare wird nicht nur an der Vielzahl der Auflagen deutlich. Der Einfluß dieser Werke zeigt sich auch in der täglichen Praxis. Der Großkommentar, 
die Kurzkommentare und die Lehrbücher, vor allem das große Lehrbuch erweisen ihren Einfluß auf die Praxis durch die Verarbeitung in den Entscheidungen und in wissenschaftlichen Abhandlungen.

Bei den Kommentaren wirken zum Teil Richter aus den oberen Gerichten mit. Dies hat den Vorteil, daß die Rechtsprechung zuverlässig und detailgetreu wiedergegeben wird. Bei den obersten Bundesrichtern ergibt sich im Fall von noch ungeklärten Rechtsfragen zugleich der Vorteil, daß man die künftige obergerichtliche Rechtsprechung zwar nicht sicher, aber doch mit einiger Wahrscheinlichkeit prognostizieren kann. Dies ist für den Absatz des Kommentarwerks sicher förderlich. Man kann aber von den Bundesrichtern nicht erwarten, daß sie sich mit ihrer eigenen Rechtsprechung kritisch auseinandersetzen. Insofern ist der Kommentar auf die bloße Informationsfunktion beschränkt. Seine Funktion, neben der zuverlässigen Information die Rechtsprechung kritisch mit eigenständigen Ideen zu begleiten, kann der Kommentar dann kaum mehr erfüllen. Diese Funktion können die Lehrbücher nach wie vor ausfüllen, wofür vor allem die große ,grüne“ Lehrbuchreihe mit ihrem größeren Seitenumfang prädestiniert ist. Die Lehrbücher üben zugleich einen prägenden Einfluß auf die Studenten aus und wirken auf diese Weise in Wissenschaft und Praxis hinein, auch wenn dies nicht immer durch Zitate nachgewiesen werden kann. Andererseits nehmen die Lehrbücher auch die Einflüsse aus der Praxis vor allem der Rechtsprechung auf und verarbeiten sie. So entsteht eine ständige Wechselwirkung zwischen Rechtsprechung, Kommentaren und Lehrbüchern, die im folgenden an einigen wesentlichen Entwicklungen im Allgemeinen Schuldrecht aufgezeigt werden soll.

\section{Weiterentwicklung des Allgemeinen Schuldrechts}

Das Allgemeine Schuldrecht bietet mit seinen generellen, abstrakt gefaßten Vorschriften die Möglichkeit, sie auf die verschiedenartigsten Fallgestaltungen anzuwenden. So ist etwa das Recht der Leistungsstörungen, einschließlich der Vorschriften über den gegenseitigen Vertrag und den Rücktritt anwendbar, gleichgültig welchen Inhalt die verletzte Pflicht aufweist. Gleiches gilt für den Annahmeverzug des Gläubigers ( $\$ 294$ BGB). Ebenso können die Vorschriften zur Bestimmung des Leistungsorts ( $\$ \$ 269,270$ BGB) und der Leistungszeit ( $\$ 271$ BGB) sowie die Vorschriften über das Zurückbehaltungsrecht (\$273 BGB) oder die Vorschriften über die Erfüllung ( $\$ 362 \mathrm{ff}$. BGB) und die Aufrechnung ( $\$ 387 \mathrm{ff}$. BGB) oder den Erlaß (§ 397 BGB) grundsätzlich ohne Rücksicht auf den Inhalt der jeweiligen Pflicht zur Anwendung gebracht werden. Auch die Abtretungsvorschriften sind aufgrund ihrer Abstraktheit grundsätzlich auf alle Forderungen anwendbar. Die Gesamtschuld setzt zwar die Gleichheit des Leistungsinteresses voraus. Ist dieses aber gegeben, dann können die Forderungen auch auf unterschiedlichem Schuldgrund beruhen. Außerdem ist eine Gesamtschuld anzunehmen, gleichgültig welchen Inhalt das Leistungsinteresse hat, sofern nur seine Identität hinsichtlich der Verpflichtungen mehrerer Schuldner gegeben ist. Deshalb steht auch die Gesamtschuld grundsätzlich für alle Pflichtinhalte zur Verfügung. Das allgemeine Schuld- 
recht ist somit prinzipiell offen für den Wandel der gesellschaftlichen und wirtschaftlichen Verhältnisse, ohne die gesetzlichen Vorschriften und rechtlichen Maßstäbe verändern zu müssen. Von diesem grundsätzlichen Ausgangspunkt gibt es jedoch Ausnahmen, die zur Weiterentwicklung des Allgemeinen Schuldrechts beitragen. Auf einige dieser Weiterentwicklungen und den Beitrag der Verlagsliteratur dazu soll im folgenden eingegangen werden.

\section{Einfluß der Grundrechte}

Einen wesentlichen Beitrag zur Fortbildung des Zivilrechts kam seit seinem Inkrafttreten dem Grundgesetz zu, das mit den in den Grundrechten enthaltenen Werten das zivilistische Denken beeinflußte. Das Allgemeine Schuldrecht bot mit $\S 242$ BGB ein Einfallstor für die grundrechtlichen Wertungen. Die Auswirkungen zeigten sich zwar nicht in der Schöpfung neuer Rechtsinstitute wie dem allgemeinen Persönlichkeitsrecht als neuem Schutzgut in $\S 823$ Abs. 1 BGB. Mit Hilfe von $\S 242$ BGB wurden aber die Inhalte von Nebenpflichten mit grundrechtlichen Wertungen aufgefüllt. Dies zeigte sich etwa im Recht der Wohnungsmiete, wo die Pflichten des Vermieters und damit zugleich die Rechte des Mieters erweitert wurden, etwa hinsichtlich des Rechts zur Aufnahme des Ehepartners oder hinsichtlich der Erweiterung des Rechts zum Empfang von Besuchern sowie hinsichtlich der Möglichkeit, über besondere Antennen ausländische Programme zu empfangen. Auch wenn diese Wertungen ihre Auswirkungen vornehmlich bei den einzelnen Vertragstypen zeigten, so stellte das Allgemeine Schuldrecht mit $\S 242$ BGB doch die Grundlagen dafür zur Verfügung.

\section{Entwicklungen im Schadensersatzrecht}

Wesentlichen Einfluß auf die Weiterentwicklung des Allgemeinen Schuldrechts nahm vor allem die wirtschaftliche Entwicklung und das Vordringen einer wirtschaftlichen Betrachtungsweise. Die gesteigerten wirtschaftlichen Bedürfnisse in dem einsetzenden Wirtschaftsaufschwung und das neue wirtschaftliche Denken beeinflußten auch die Rechtswissenschaft und die Rechtspraxis im Zivilrecht. Im Allgemeinen Schuldrecht zeigte sich dies beispielhaft vor allem im Schadensersatzrecht.

\section{Ausdehnung des immateriellen Schadens}

Galt es bei Inkrafttreten des BGB noch als unschicklich, aus der Verletzung immaterieller Werte Kapital zu schlagen, so forderte doch ausgeprägtes wirtschaftliches Denken bald seinen Tribut im Zivilrecht. Die Vorschrift des $§ 253$ BGB, die den Ersatz des immateriellen Schadens ausschloß, außer wenn eine gesetzliche Bestimmung einen solchen Ersatz ausdrücklich vorsah, erwies sich schon bald vor allem bei Verletzungen des Persönlichkeitsrechts als zu eng. Rücksichtslose Medien, die aus der Verletzung des Namensrechts, des Rechts am Bild und der Privatrechtssphäre sich den Absatz ihrer Produkte und Gewinn versprachen, muß- 
ten in die Schranken verwiesen werden. Dies konnte, sofern Unterlassungsansprüche nicht mehr zum Zug kommen konnten, vor allem durch eine Art Gewinnabschöpfung mit Hilfe von Schadensersatzansprüchen gelingen. Zunächst wurden hierzu die Möglichkeiten ausgeschöpft, die das Recht des materiellen Vermögensschadens mit dem entgangenen Gewinn bot, sei es in Form konkret nachgewiesenen Einnahmeausfalls oder mit Hilfe fiktiver Lizenzeinnahmen. Vielfach war aber mit dem Gedanken des materiellen Vermögensschadens nicht zu helfen, weil der Verletzte gar nicht die Absicht hatte, mit der Vermarktung seines Persönlichkeitsrechts Gewinn zu machen. Das Hindernis des $\S 253$ BGB umschiffte die Rechtsprechung ${ }^{1}$ dabei mit einer analogen Anwendung des Schmerzensgeldanspruchs, den der ehemalige $\$ 847$ BGB nur für Körperverletzungen und Freiheitsentziehungen vorsah, nicht aber für Verletzungen des Persönlichkeitsrechts. Die ausdehnende Anwendung des Schmerzensgeldanspruchs war neben den erwähnten ökonomischen Überlegungen auch wesentlich von dem Bedürfnis nach möglichst effektivem Schutz des aus Art. 1 und 2 GG abgeleiteten Persönlichkeitsrechts bestimmt. Deshalb wird der immaterielle Schadensersatz bei Verletzung des Persönlichkeitsrechts jetzt unmittelbar aus $\S 823$ BGB mit Art. 1 und 2 GG hergeleitet. ${ }^{2}$

Seit dem 1. August 2002 ist der Ersatz des immateriellen Schadens aus der Verankerung im speziellen Rechtsgebiet der unerlaubten Handlung herausgelöst und im Allgemeinen Schuldrecht in Form einer Erweiterung des $§ 253$ BGB um einen Absatz 2 angesiedelt worden. Der mit dem Allgemeinen Schuldrecht verbundenen Vorstellung, damit alle Fälle des immateriellen Schadens zu erfassen, ist der Gesetzgeber aber nicht gerecht geworden. $§ 253$ BGB bezieht sich neben den bisher von $\S 847$ BGB a.F. unmittelbar erfaßten Ansprüchen aus unerlaubter Handlung zwar auch auf Ansprüche aus Gefährdungshaftung, für die bisher spezielle Regelungen bestanden. Ebenso können Schmerzensgeldansprüche wegen vertraglicher Pflichtverletzung auf $\S 253$ Abs. 2 BGB gestützt werden. ${ }^{3}$ Die Spezialvorschrift des $\S 651 \mathrm{f} \mathrm{BGB}$ ist deshalb aber nicht entfallen, weil $\S 253$ Abs. 2 BGB wie $\S 847$ BGB a.F. nur die Verletzung des Körpers, der Gesundheit, der Freiheit und neu eingefügt auch der Verletzung der sexuellen Freiheit, nicht aber die Beeinträchtigung der Urlaubsfreude umfaßt. ${ }^{4}$ In $\S 253$ Abs. 2 BGB nicht geregelt ist auch der Schadensersatz wegen Verletzung des Persönlichkeitsrechts. Obwohl hierzu eine gefestigte Rechtsprechung vorliegt, hat sich der Gesetzgeber nicht zu einer Aufnahme des Schutzguts „Persönlichkeitsrecht“" in $§ 253$ Abs. 2 BGB entschließen können.

Dies kann teilweise damit gerechtfertigt werden, daß bei der Bemessung des Schmerzensgeldes für Verletzungen des Persönlichkeitsrechts aus Gründen der Prävention auch der erzielte oder auch nur angestrebte Gewinn zu berücksichtigen ist. ${ }^{5}$ Es bleibt aber insgesamt doch unbefriedigend und entspricht kaum der Kon-

${ }^{1}$ Ausgangsentscheidung war $B G H Z$ 26, 361.

2 BGHZ 128, 1, 15; 143, 214, 218.

${ }^{3}$ S. dazu etwa Palandt-Heinrichs, BGB, 66. Aufl. 2007, § 253 Rn. 8.

${ }^{4} \mathrm{Zu}$ zahlreichen verbleibenden Spezialvorschriften s. MünchKommBGB-Oetker, BGB, 4. Aufl. 2003, § 253 Rn. 1 und 2; Bamberger/Roth/Spindler, BGB, 1. Aufl. 2003, § 253 Rn. 9f.

${ }^{5}$ BGHZ 128, 1; Palandt-Heinrichs (Fn. 3), § 253 Rn. 10. 
zeption eines Allgemeinen Teils und einer umfassenden Regelung des immateriellen Schadens. In der Entscheidung des Gesetzgebers, das Persönlichkeitsrecht nicht als Schutzgut in $\S 253$ Abs. 2 BGB aufzunehmen, liegt aber keine Ablehnung eines Ersatzes für immaterielle Schäden. Angesichts der ausdrücklichen Entscheidung des Gesetzgebers gegen eine Regelung des immateriellen Schadens bei Verletzung des Persönlichkeitsrechts ${ }^{6}$ ist allerdings auch eine analoge Anwendung des $\S 253$ Abs. 2 BGB nicht zu rechtfertigen ${ }^{7}$. Der Ersatz immateriellen Schadens bei Verletzung des Persönlichkeitsrechts muß deshalb nach wie vor auf $\S 823$ BGB mit Art. 1 und 2 GG gestützt werden. ${ }^{8}$ Jedoch sollte der Präventionsgedanke, der bei der Bemessung des immateriellen Schadens im Falle der Verletzung des Persönlichkeitsrechts zur Anwendung kommt, auch beim Schmerzensgeld im Rahmen von $§ 253$ Abs. 2 BGB Berücksichtigung finden. ${ }^{9}$

\section{Kommerzialisierung beim materiellen Schaden?}

Die verstärkte wirtschaftliche Betrachtungsweise hat auch zu einer Ausdehnung und Erweiterung des materiellen Schadensbegriffs geführt. Dabei stand einige Zeit der Kommerzialisierungsgedanke im Vordergrund. Danach war ein Schaden dann anzunehmen, wenn ein Gut beeinträchtigt wurde, das gegen Entgelt erworben werden konnte. Nach diesem Kommerzialisierungsgedanken wurden im Jahre 1974 etwa Aufwendungen für nutzlos aufgewendete Urlaubszeit als Schaden angesehen, wenn die Reiseleistung so schwerwiegende Mängel aufwies, daß der dadurch entstandene Schaden mit einer Herabsetzung des Reisepreises nicht ausgeglichen werden konnte. ${ }^{10}$ Diese Frage ist jetzt, soweit es die Erbringung mangelhafter Leistungen im Rahmen eines Reisevertrags betrifft, durch $\S 651 \mathrm{f}$ Abs. 2 BGB positiv rechtlich geregelt. Dabei hat der Gesetzgeber die Ablehnung eines Vermögensschadens bestätigt und mit der Zubilligung einer angemessenen Entschädigung einen Anspruch auf Ersatz des immateriellen Schadens gewährt.

Auch die Einordnung des Nutzungsausfalls eines Kraftfahrzeugs als Schaden ${ }^{11}$ wurde zuvor schon im Jahre 1963 auf den Kommerzialisierungsgedanken gestützt. ${ }^{12}$ Dem Ausfall der Nutzungsmöglichkeit eines Kraftfahrzeugs, dem der Verkehr einen selbständigen wirtschaftlichen Wert beimesse, stellte die Rechtsprechung andererseits Nutzungsmöglichkeiten gegenüber, die nach der Verkehrsauffassung als Luxus (so für die Nichtbenutzbarkeit eines Pelzmantels ${ }^{13}$ oder eines Wohnwagens ${ }^{14}$ ), als bloße Liebhaberei (so die Benutzung des eigenen Schwimmbads ${ }^{15}$ ) oder als bloßes Mittel zur Freizeitgestaltung (so für die Nutzungsmöglich-

${ }^{6}$ BT-Drucks. 14/7752, S. 24 f., 49 f., 55.

7 MünchKommBGB-Oetker (Fn. 4), § 253 Rn 27.

8 Allg. Meinung: MünchKommBGB-Oetker (Fn. 4), § 253 Rn. 27; Palandt-Heinrichs (Fn. 3), $\S 253$ Rn. 10; Brox/Walker, Allgemeines Schuldrecht, 32. Aufl. 2006, § 29 Rn. 6.

9 S. Bamberger/Roth/Spindler (Fn. 4), § 253 Rn. 18.

${ }_{10}$ BGHZ 63, 98, 106; zuvor BGH NJW 1956, 1235 (Seereise).

${ }_{11}$ BGHZ 40, 345, 347; 45, 212, 218; 56, 214; 65, 170.

12 So ausdrücklich BGHZ 63, 98, 101 f., 104.

13 BGHZ 63, 393, 398.

14 BGHZ 86, 128, 133.

15 BGHZ 76, 179, 187. 
keit eines Motorsportbootes ${ }^{16}$ ) zu werten seien und keinen Ersatz für Nutzungsausfall rechtfertigten. Abgelehnt wurde auch ein Ersatz für den Wegfall der Arbeitskraft ohne konkreten Nachweis eines Einnahmeausfalls. ${ }^{17}$

Die Unsicherheiten, die durch diese Rechtsprechung geschaffen wurden, führten zur Vorlage an den Großen Senat für Zivilsachen, der an Hand des Nutzungsausfalls eines Eigenheims im Jahre 1986 entschied, daß über die am bloßen Geldwert orientierte Differenzmethode hinaus eine - wirtschaftlich - wertende Betrachtung stattzufinden habe. ${ }^{18}$ Dies führe dazu, daß ein Vermögensschaden nicht nur dann zu bejahen sei, wenn eine für erwerbswirtschaftliche Zwecke bestimmte Sache nicht mehr genutzt werden könne und dadurch ein Gewinnausfall entstehe. Vielmehr müsse ein Vermögensschaden auch dann angenommen werden, wenn ein Wirtschaftsgut von zentraler Bedeutung, wie das Eigenheim, auf dessen ständige Verfügbarkeit für die eigene Lebenshaltung der Geschädigte typischerweise angewiesen ist, nicht mehr zur Eigennutzung zur Verfügung stehe. ${ }^{19}$

Die grundsätzliche Entscheidung allgemein für oder gegen den Kommerzialisierungsgedanken, die eigentlich vom Großen Senat für Zivilsachen erwartet worden war, blieb aus. Statt dessen legte er sich auf eine fallbezogene Lösung fest. Diese ging aus von der Entschädigung für den Nutzungsausfall von Kraftfahrzeugen, der sich in der Schadens- und Versicherungspraxis durchgesetzt hatte und aus Gründen der Rechtssicherheit nicht mehr zurückgedreht werden konnte. Auf dieser Grundlage lag es nahe, den Nutzungsausfall eines Kraftfahrzeugs nicht schwerer zu bewerten als den Nutzungsausfall des Eigenheims. Dies kleidete der Große Senat für Zivilsachen in die Formel, daß für den Nutzungsausfall von solchen Sachen Ersatz zu leisten sei, ,auf deren ständige Verfügbarkeit die eigenwirtschaftliche Lebenshaltung typischerweise angewiesen ist." Darin lag im Grunde eine Ablehnung des allgemeinen Kommerzialisierungsgedankens.

Der Ablehnung des Kommerzialisierungsgedankens können auch Entscheidungen zugerechnet werden, welche die Aufopferung von Freizeit, sei es zwecks Abwicklung des eigenen Schadensfalles ${ }^{20}$, sei es, daß Zeit für andere Geschädigte (Pflege und Krankenhausbesuche des geschädigten Kindes) aufgewendet wurde, nicht als ersatzfähigen Vermögensschaden ansehen ${ }^{21}$ und damit dem in $§ 253$ BGB verankerten Gedanken des nicht durch Geldersatz auszugleichenden immateriellen Schadens zur Geltung verhelfen. In dieser Haltung ist die Rechtsprechung konsequent geblieben und wurde darin auch durch die Literatur bestärkt. ${ }^{22}$ Die gegenteilige Auffassung von Grunsky im Münchener Kommentar, ${ }^{23}$ wonach der Verlust an Freizeit identisch sei mit dem Verlust der Chance, Geld zu verdienen, hat sich

\footnotetext{
${ }^{16}$ BGHZ 89, 6064.

17 BGHZ 54, 45.

18 Zuvor schon $B G H Z$ 45, 212, 218 unter Hinweis auch auf das Lehrbuch von Larenz.

${ }_{19}$ BGHZ 98, 212, 220, 222; daran anknüpfend BGHZ 117, 260, 262.

${ }^{20}$ Dazu BGHZ 66, 112; 75, 230, 231 ff. (Ladendiebstahl); 127, 350; BGH NJW 1996, 922.

${ }^{21}$ BGHZ 106, 28, 32.

22 S. etwa Larenz, Lehrbuch des Schuldrechts, Band I, Allgemeiner Teil, 14. Aufl. 1987, § 29 II d.

${ }^{23}$ MünchKommBGB-Grunsky, 3. Aufl., vor § 249 Rn. 30.
} 
nicht durchsetzen können. Dagegen fallen Unkosten, die zur Wiedererlangung von entwendeten Sachen aufgewandt worden sind, wie etwa eine Fangprämie beim Ladendiebstahl, unter den ersatzfähigen Schaden ${ }^{24}$, während Kosten, die allgemein zum Schutz des Eigentums vor Diebstahl aufgewendet werden, vom Geschädigten selbst zu tragen sind ${ }^{25}$.

Die Ablehnung des allgemeinen Kommerzialisierungsgedankens durch den $B G H$ ist sicher auch der ablehnenden Haltung von Larenz zuzuschreiben, die dieser schon 1963 zum Ausdruck gebracht hatte ${ }^{26}$ und diese Auffassung in sein Lehrbuch zum Schuldrecht übernahm. Das Lehrbuch war 1953 in erster Auflage im Verlag C.H. Beck erschienen. Es war bei der Entscheidung aus dem Jahre 1974 als alleiniges Lehrbuch in 10. Auflage erwähnt ${ }^{27}$ und in der Entscheidung des Großen Senats in Zivilsachen in 13. Auflage 1982 neben anderen Lehrbüchern zitiert.

\section{Vordringen des normativen Schadensbegriffs}

Schon vor der Ablehnung einer allgemeinen Kommerzialisierung des Schadens hatte sich die Erkenntnis durchgesetzt, daß der Begriff des Vermögensschadens nicht allein mit der vornehmlich am Geldwert orientierten Differenzmethode erklärt werden kann. Die Differenzhypothese bildet zwar den Ausgangspunkt und den Grundstock des Vermögensschadens. Sie bedarf aber der Korrektur durch normative Erwägungen. Es ist deshalb von einem normativen Schadensbegriff auszugehen. Nicht der Schaden an sich ist zu bestimmen, sondern der rechtlich ersatzfähige Schaden. Zwar waren einzelne normative Erwägungen wie etwa die Versagung der Vorteilsausgleichung schon zuvor etwa vom $R G^{28}$ und von Anfang an vom $B G H^{29}$ berücksichtigt worden. Als allgemein gültige Erkenntnis hatte sich der normative Schadensbegriff jedoch erst im Laufe der 60er Jahre des 20. Jahrhunderts durchgesetzt, wie Heinrichs im Kommentar von Palandt feststellt. ${ }^{30}$ Die Feststellung im Kommentar von Palandt, daß der umfassend normativ zu verstehende Schadensbegriff eine allgemein gültige Erkenntnis sei, beeinflußt wesentlich die Praxis, weil der Beck-Verlag diesen Kommentar mit seinem jährlichen Erscheinen seit der 7. Auflage 1949 zum leitenden Kommentar für die Praxis gemacht hat. Eine positive Bestimmung dessen, was der normative Schaden im Einzelfall ist, wird damit jedoch nicht geleistet. Dazu müssen verschiedene normative Erwägungen berücksichtigt werden, die in unterschiedlichen Fallgruppen und richterrechtlichen Rechtssätzen konkretisiert und verfeinert worden sind. So ist neben der Versagung der Vorteilsausgleichung ${ }^{31}$ etwa auch die Frage, wann für nutzlos gewordene Aufwendungen Schadensersatz zu leisten ist, nach normativen

\footnotetext{
${ }^{24}$ BGHZ 75, 230, 235 ff. unter Hinweis auch auf Larenz (Fn. 22), § 29 IIf. und PalandtHeinrichs (Fn. 3), § 249 Anm. 3b und Palandt-Thomas, § 823 Anm. 12 a.

${ }_{25}$ BGHZ 75, 230, 237 unter Hinweis auch auf Larenz (Fn. 22), § 29 II f.

26 Larenz, VersR 1963, 312; ders. (Fn. 22) § 29 IIf.

27 BGHZ 63, 98, 106S; s. auch BGHZ 76, 179, 184.

28 S. etwa schon RGZ 82, 192; 92, 57, 146, 278.

${ }^{29}$ S. BGHZ 8, 329.

30 Palandt-Heinrichs (Fn. 3), Vorbem. vor $§ 249$ Rn. 13.

31 BGHZ 7, 30, 21; 112; 43, 378, 381.
} 
Gesichtspunkten zu entscheiden. Auch hierbei bezieht sich der BGH auf das Lehrbuch von Larenz. ${ }^{32}$

\section{Schutzzweck der Norm}

Eine weitere wichtige Entwicklung, die in der Literatur ihren Anfang nahm und die im Jahre 1958 in die Rechtsprechung Eingang fand, ist die Lehre vom Schutzzweck der Norm. ${ }^{33}$ Bei dieser normativen Durchdringung des Schadensersatzrechts geht es nicht wie beim Kommerzialisierungsgedanken um die Abgrenzung von materiellem und immateriellem Schaden, sondern um den einer Pflichtverletzung zurechenbaren Schaden. Für diese Zurechenbarkeit war lange Zeit allein eine Kausalbetrachtung maßgebend, die in der abgemilderten Form der Adäquanzlehre alle kausal auf die Pflichtverletzung rückführbaren Schäden in die Ersatzpflicht einbezog, sofern es sich nicht um Schäden handelte, die nach der Lebenserfahrung vernünftigerweise nicht in Betracht gezogen werden mußten. Die Anwendung dieser Formel bewirkte lediglich in Extremfällen eine Begrenzung des zu ersetzenden Schadens, so etwa wenn eine geringfügige Ehrverletzung zu einer Gehirnblutung führt ${ }^{34}$ oder die Auseinandersetzung und Aufregung über einen Verkehrsunfall zu einem Schlaganfall ${ }^{35}$. Über die ungewöhnlichen Fallgestaltungen, die in der Praxis der Gerichte zu entscheiden waren, kann man sich im Kommentar von Palandt einen raschen Überblick verschaffen. ${ }^{36}$ Die Adäquanzlehre bewirkte jedoch insgesamt keine zufriedenstellende Begrenzung der Haftung und konnte insbesondere im Rahmen der haftungsausfüllenden Kausalität, in der das Korrektiv des Verschuldens nicht eingreift, zu einer allzu weitgehenden Zurechnung von Schäden führen. Die Lehre vom Schutzzweck der Norm konnte hier zu sinnvollen Eingrenzungen beitragen, indem sie als ersatzfähig nur solche Schäden ansah, vor deren Eintritt die verletzte Norm schützen sollte. Der eingetretene Schaden mußte im Bereich der Gefahren liegen, zu deren Vermeidung die verletzte Norm oder Pflicht vorgesehen wurde.

Die Haftpflichtbegrenzung nach dem Schutzzweck der Norm liegt vor allem nahe bei Anspruchsgrundlagen, die - wie $§ 823$ Abs. 2 BGB - auf die Verletzung eines Schutzgesetzes oder wie in $\S 839$ BGB auf die Verletzung einer drittschützenden Amtspflicht abstellen. ${ }^{37}$ Sie hat aber darüber hinaus allgemeine Bedeutung und ist insbesondere auch bei Ansprüchen aus $\S 823$ Abs. 1 BGB anzuwenden ${ }^{38}$ sowie auf Schäden aus vertraglichen Pflichtverletzungen ${ }^{39}$.

Im Ausgangsfall zur Anwendung der Normzwecklehre auf $\S 823$ Abs. 1 BGB hatte der BGH darüber zu entscheiden, ob der an einem Verkehrsunfall Beteiligte,

\footnotetext{
32 S. etwa $B G H Z$ 55, 146, 151; 65, 170, 181; Larenz (Fn. 22), § 29 II f.

33 Als Begründer der Lehre gilt Rabel, Das Recht des Warenkaufs, Band I, 1936, S. $495 \mathrm{ff}$.

34 BGH NJW 1976, 1143.

35 BGHZ 107, 359, 361.

36 S. Palandt-Heinrichs (Fn. 3), Vorb. vor $§ 249$ Rn. 68.

${ }^{37}$ S. etwa $B G H Z$ 39, 365; 90, 310, $312=$ NJW 1984, 2691; BGH NJW 1990, 2615, 2616.

${ }^{38}$ S. dazu $B G H Z$ 27, 138; BGHZ 32, 205; zur ärztlichen Aufklärung etwa BGH NJW 1991, 2346, 2347.

39 BGH NJW 1990, 2057; BGH NJW 1995, 451; BGH NJW 1997, 2947.
} 
aber im späteren Strafverfahren Freigesprochene Ersatz seiner Verteidigungskosten im Strafverfahren erlangen könne. ${ }^{40}$ Der $B G H$ ging unter Hinweis auch auf das Lehrbuch von Larenz davon aus, daß mit Hilfe der adäquaten Kausalität der Schaden nicht immer sachgerecht begrenzt werden könne und stützte sich deshalb auch im Rahmen von $\S 823$ Abs. 1 BGB auf die Normzwecklehre, lehnte aber einen Ersatz im konkreten Fall ab, weil der Schaden nicht in den Schutzbereich des $§ 823$ Abs. 1 BGB falle.

Schutzzweckerwägungen hat der $B G H$ im Bereich der vertraglichen Schadensersatzansprüche zunächst durch Anwendung von § 254 BGB Geltung zu verschaffen versucht. ${ }^{41}$ In einem späteren Fall hat er aber auch bei vertraglichen Schadensersatzansprüchen sich ausdrücklich auf die Schutzzwecklehre gestützt. In diesem Fall berief sich der Kläger darauf, über steuerliche Risiken nicht aufgeklärt worden zu sein. Obwohl diese Risiken im Ergebnis nicht eintraten, verlangte der Kläger Schadensersatz mit der Begründung, bei Kenntnis der steuerlichen Risiken, hätte er eine andere ertragreichere Investition getätigt. Der $B G H$ versagte den Schadensersatzanspruch, weil die steuerliche Beratung den Kläger nur vor steuerlichen Schäden, nicht aber vor Schäden aus einer wirtschaftlich verfehlten Investitionsentscheidung schützen sollte. ${ }^{42}$

Zum Eingang der Lehre vom Schutzzweck der Norm in die Rechtsprechung haben die Lehrbücher und Kommentare des Beck-Verlags zwar nicht unmittelbar beigetragen. Sie haben diese Lehre aber alsbald aufgenommen und ausgebaut. Auf diese Weise haben sie die Rechtsprechung auf ihrem Weg bestärkt. Dies zeigen insbesondere die in den Entscheidungen verarbeiteten Zitate, so etwa der Hinweis auf Grunsky im Münchener Kommentar in der Entscheidung BGH NJW 1990, 2057, 2059, in der es um die Anwendung der Schutzzwecklehre auf vertragliche Pflichtverletzungen ging. Diese Einflüsse der Literatur wirken nach, auch wenn Literatur üblicherweise nur in der ersten Entscheidung, die sich mit dem Problem befaßt, verarbeitet wird und die nachfolgenden Entscheidungen nur noch die vorausgegangenen Urteile zitieren.

Mit normativen Erwägungen ist auch die Ablehnung von Schadensersatzansprüchen in den Fällen der Rentenneurose zu rechtfertigen. Hatte die Rechtsprechung des RG hier ursprünglich noch Schadensersatzansprüche gewährt, ${ }^{43}$ weil ein Kausalzusammenhang nicht verneint werden konnte, so boten dem $B G H$ später Normzwecküberlegungen die Grundlage für die Ablehnung von Schadensersatzansprüchen, weil es dem Zweck des Schadensersatzes widerspricht, wenn gerade durch die Schadensersatzleistung die Wiederherstellung des gesunden Zustands verhindert wird ${ }^{44}$.

Weder mit der Adäquanzlehre noch mit der Normzwecklehre sind die sogenannten Verfolgungsfälle zu lösen, in denen etwa Polizisten oder sonstige Personen einen Täter verfolgen und dabei verunglücken und zu Schaden kommen. Davon

40 BGHZ 27, 137.

41 S. etwa $B G H Z 57,137,142$.

42 BGH NJW 1990, 2057, 2058.

43 RGZ 75, 19; 159, 257, 262.

${ }^{44}$ BGHZ 20, 142; BGH NJW 1979, 1936. 
geht der $B G H$ in seiner grundlegenden Entscheidung unter Hinweis auf das Lehrbuch von Larenz zu Recht aus. ${ }^{45}$ Es geht aber jeweils um Fragen der Schadenszurechnung, die nicht mit reinen Kausalbetrachtungen gelöst werden können, sondern der normativen Bewertung bedürfen. Die Rechtsprechung hat diese Fälle mit dem Kriterium gelöst, ob sich der Geschädigte zur Verfolgung herausgefordert fühlen durfte. ${ }^{46}$ Darauf, daß dieses Kriterium nicht nur bei der haftungsausfüllenden Kausalität, sondern auch im Rahmen der haftungsbegründenden Zurechnung zur Anwendung kommen kann, bezieht sich der $B G H$ auf das Lehrbuch von Laren $z^{47}$. Mit dem Merkmal der Herausforderung wird im Ergebnis geprüft, ob der Entschluß zur Verfolgung vernünftig war oder nicht. ${ }^{48}$ Das Abstellen auf das Merkmal, ob sich der Geschädigte zu dem Verhalten, durch das er sich selbst schädigte, herausgefordert fühlen durfte, ist ein sachgerechtes Kriterium der Schadenszurechnung auch in anderen Fällen der Selbstschädigung, wenn der Geschädigte das rechtswidrige Verhalten eines anderen als Anlaß zum Eingreifen nimmt und sich dadurch selbst schädigt. ${ }^{49}$

So richtig und überzeugend in manchen Fällen die Begründung mit der Normzwecklehre sein mag, so hinterläßt die Rechtfertigung mit normativen Bewertungen in anderen Fällen doch Unsicherheiten und Zweifel. Dies führt dazu, daß nicht nur in den Kommentaren, sondern auch in den Lehrbüchern, von denen man eine systematische Verarbeitung und Stellungnahme erwarten würde, nur noch die entschiedenen Einzelfälle ohne eigene Stellungnahme aufgeführt wer$\operatorname{den}^{50}$ oder aber es kommt zu gegensätzlichen Bewertungen, wie etwa bei der Frage, ob bei Verletzung eines Bluters ein besonders hoher Schaden zu ersetzen sei ${ }^{51}$. Mit dieser Fallgestaltung rechtfertigen Brox/Walker die Anwendung der Adäquanzlehre neben der Lehre vom Schutzzweck der Norm ${ }^{52}$ und während der $B G H$ die Ersatzfähigkeit des Schadens aufgrund eines Schlaganfalls als Folge der Aufregung über einen Verkehrsunfall ${ }^{53}$ mit der Normzwecklehre ablehnt, wollen andere die adäquate Kausalität verneinen ${ }^{54}$. Die Anwendung der Adäquanzlehre neben der Normzwecklehre kann damit zur Absicherung von Ergebnissen beitragen, mögen sie auch unterschiedlich begründet werden. Dagegen verursacht der mit der Normzwecklehre geschaffene Bewertungsspielraum zusätzliche Rechtsunsicherheit, die die Vorhersage des Ergebnisses von Gerichtsurteilen kaum mehr zuverlässig ermöglicht und damit den Gerichten umso mehr Arbeit beschert, weil jede Partei noch auf eine ihr günstige Entscheidung hoffen kann. Dies ist

\footnotetext{
45 BGHZ 57, 25, 29; Larenz (Fn. 22), § 27 III b 4.

46 BGH NJW 1964, 1363, 1364; BGHZ 57, 25; 63, 189; BGH VersR 1991, 111.

47 BGHZ 57, 25, 30; Larenz (Fn. 22), § 27 III b 5.

48 So zu Recht Medicus, Schuldrecht I, Allgemeiner Teil, 17. Aufl. 2006, Rn. 602.

49 S. MünchKommBGB-Oetker (Fn. 4), § 249 Rn. 171.

50 S. etwa Medicus (Fn. 48), Rn. 601 f.; Larenz (Fn. 22), S. 545 zur Rentenneurose.

51 Vgl. einerseits Palandt-Heinrichs (Fn. 3), Vorb. vor $§ 249$ Rn. 67 bejahend; andererseits Brox/Walker (Fn. 8), § 30 Rn. 15 verneinend.

52 Brox/Walker (Fn. 8), § 30 Rn. 15; dafür auch Medicus (Fn. 48), Rn. 600; Larenz (Fn. 22), jedoch differenzierend zwischen Verschuldenshaftung und Gefährdungshaftung S. 441, 456.

53 BGHZ 107, 359.

${ }^{54}$ Medicus (Fn. 48), Rn. 600; von Bar, JZ 1989, 1071; Lipp, JuS 1991, 809.
} 
ein Charakteristikum der derzeitigen Rechtsentwicklung, worin der alte Konflikt zwischen Rechtssicherheit und Einzelfallgerechtigkeit zum Ausdruck kommt, ${ }^{55}$ der aber derzeit allzu einseitig zugunsten der Einzelfallgerechtigkeit gehandhabt wird.

\section{Vertrag mit Schutzwirkung für Dritte}

Zum Schadensersatzrecht gehören nicht nur die Bestimmung des Schadensbegriffs und die Zurechnung von Schäden, sondern auch die Ausformung und Präzisierung von Anspruchsgrundlagen. Diese Anspruchsgrundlagen finden sich häufig im Besonderen Teil des Schuldrechts, vor allem im Recht der unerlaubten Handlung und bei den einzelnen Vertragstypen. Das allgemeine Recht der Leistungsstörungen in den $\$ \S 280 \mathrm{ff}$. BGB hat bis zur Schuldrechtsreform im Jahre 2002 keine wesentlichen Veränderungen und neuen Entwicklungen aufzuweisen. Eine maßgebliche Neuerung und Ausweitung der Haftungsgrundlagen, die sich in der zweiten Hälfte des 20. Jahrhunderts entwickelt hat, stellt aber die Figur des Vertrags mit Schutzwirkung zugunsten Dritter dar. Sie war z. B. in der 18. Auflage des Kommentars von Palandt aus dem Jahre 1959 noch nicht als Stichwort zu finden. Auch das große Lehrbuch zum Schuldrecht von Enneccerus/Lehmann behandelt sie in der 14. Auflage von 1954 noch nicht besonders. Zwar sind der Sache nach schon in der Rechtsprechung des RG Dritte in den Schutzbereich der Sorgfaltspflichten eines Vertrags einbezogen worden. ${ }^{56}$ Als eigenständige Rechtsfigur wurde diese Sonderform des Vertrags zugunsten Dritter aber erst Ende der 50er Jahre herausgearbeitet ${ }^{57}$ und in die Lehrbücher aufgenommen. Dabei übernahm das Lehrbuch von Larenz zum Allgemeinen Teil des Schuldrechts eine gewisse Vorreiterrolle, indem er den Vertrag mit Schutzwirkung für Dritte klar vom Vertrag zugunsten Dritter als dessen schwächere Form abhob. ${ }^{58}$ Die Rechtsprechung, die zuvor noch ohne diese Unterscheidung arbeitete, übernahm bald darauf diese Einordnung. ${ }^{59}$

Anfangs war der Vertrag mit Schutzwirkung zugunsten Dritter auf solche Dritte beschränkt, denen gegenüber einer Partei besondere Fürsorgepflichten oblagen und diese Partei für das Wohl und Wehe des Dritten zu sorgen hatte, wie etwa die Eltern gegenüber ihren Kindern oder wie der Arbeitgeber gegenüber seinen Arbeitnehmern. Diese „Wohl und Wehe“-Formel begrenzte lange Zeit den Kreis der in den Schutzbereich einbezogenen Dritten. Erst als im Zuge der Suche nach einer Rechtsgrundlage für diese Figur die Zuordnung zu §328 BGB zweifelhaft wurde und die Rechtsgrundlage in einer ergänzenden Vertragsauslegung nach $\S \S 157,242$ BGB gesehen wurde, ${ }^{60}$ war auch der Weg bereitet für eine Erweiterung des geschützten Personenkreises. So hat der BGH etwa Schutzpflichten gegen-

55 S. dazu auch Summum ius, summa iniuria.

56 S. RGZ 91, 24; 102, 232; 127, 222.

57 S. Gernhuber in FS für Nikisch 1958, S. 249 ff.; Larenz, NJW 1960, 78.

58 Larenz (Fn. 22), § 17 II.

59 BGH NJW 1959, 1676; BGH NJW 1960, 77; BGHZ 49, 353.

60 S. dazu BGHZ 56, 273; BGH NJW 1996, 2927; BGH NJW 1998, 1948; dagegen aber Larenz (Fn. 22), § 17 II im Anschluß an Gernhuber in FS für Nikisch 1958, S. 249, 265. 
über dem Teilnehmer am Lastschriftverfahren anerkannt ${ }^{61}$ ebenso beim Scheckinkasso $^{62}$. Besonders häufig stellt sich die Frage, inwieweit Dritte in den Schutzbereich eines Gutachtervertrags einbezogen sind, wenn der Gutachter weiß, daß das Gutachten auch Dritten vorgelegt wird. Der BGH neigt in diesen Fällen dazu, den Dritten in den Schutzbereich des Vertrags einzubeziehen, so z. B. im Falle eines Bewertungsgutachtens für eine Immobilie, das vom Verkäufer in Auftrag gegeben worden ist, um es beim Verkauf dem Käufer vorzulegen. ${ }^{63}$ Auch Kapitalanleger werden in den Schutzbereich eines Vertrags einbezogen, durch den der Auftraggeber einen Wirtschaftsprüfer mit der Prüfung eines Werbeprospekts für die Beteiligung an einer Kapitalanlagegesellschaft beauftragt hat.

Die Einbeziehung Dritter in den Schutzbereich eines Vertrags wird vor allem angestrebt wegen der Vorteile, die die vertragliche Haftung gegenüber der Haftung aus unerlaubter Handlung hat. Dazu gehört vor allem, daß die vertragliche Haftung nicht den Eingriff in ein absolutes Recht oder die Verletzung eines Schutzgesetzes erfordert. Auch die Anwendung von $\S 278$ BGB und die Beweislastumkehr hinsichtlich des Verschuldens ist bei der vertraglichen Haftung vorteilhaft.

In Auswertung der Rechtsprechung hat das Schriftum auch die Voraussetzungen des Vertrags mit Schutzwirkung für Dritte herausgearbeitet. ${ }^{64}$ Es muß eine Nähebeziehung des Dritten zur vertraglichen Leistung bestehen, so daß der Dritte den Gefahren der Leistung in ähnlicher Weise ausgesetzt ist wie der Gläubiger (Leistungsnähe $\left.{ }^{65}\right)$. Der Gläubiger muß weiterhin ein Interesse an der Einbeziehung des Dritten haben, was in den „Wohl und Wehe“-Fällen zutrifft, in anderen Fällen wie etwa den Gutachterfällen aber manchmal nur schwer festzustellen ist. Dann genügt, daß der Dritte mit Kenntnis des Gläubigers im Vertrauen auf die vertragliche Leistung Dispositionen trifft. ${ }^{66}$ Der Kreis der Dritten muß überschaubar sein, der Dritte muß aber nicht in Person bekannt sein. Auf die Überschaubarkeit des Kreises der Dritten verzichtet der BGH in neueren Urteilen, wenn der Schaden unabhängig von der Zahl der Dritten gleich bleibt. ${ }^{67}$ Weiter wird vorausgesetzt, daß der Schuldner die Leistungsnähe und das Einbeziehungsinteresse bzw. das Dispositionsvertrauen des Dritten bei Abschluß des Vertrags erkennen kann. ${ }^{68}$ Schließlich muß der Dritte schutzbedürftig sein und darf nicht selbst durch einen vertraglichen Anspruch abgesichert sein. ${ }^{69}$ Das Schutzbedürfnis des Dritten bejaht der $B G H$ in seiner jüngsten Rechtsprechung schon, wenn eigene Ansprüche des

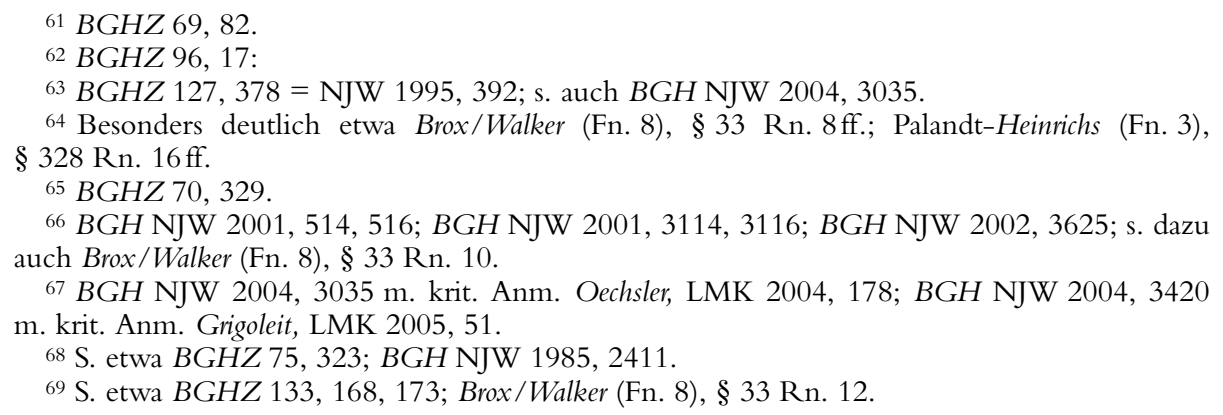


Anlegers aus Prospekthaftung einer kürzeren Verjährung unterliegen als Ansprüche aus Vertrag mit Schutzwirkung für Dritte. ${ }^{70}$ Die Reaktion der Autoren der Lehrbücher und Kommentare des Verlags auf diese Ausweitung des Vertrags mit Schutzwirkung für Dritte darf man mit Spannung erwarten. Gottwald im Münchener Kommentar ${ }^{71}$ bejaht die Schutzbedürftigkeit, wenn der eigene Anspruch des Dritten andere Voraussetzungen hat, und kommt damit der Rechtsprechung des $B G H$ sehr nahe. $\mathrm{Ob}$ abweichende Stimmen den auf Stetigkeit seiner Rechtsprechung bedachten $B G H \mathrm{zu}$ einer Änderung veranlassen oder doch bei der weiteren Aufweichung der Voraussetzungen mäßigen werden, bleibt abzuwarten.

Eine noch nicht abschließend geklärte Frage ist die Abgrenzung des Vertrags mit Schutzwirkung für Dritte gegenüber der Drittschadensliquidation. Als maßgeblicher Gesichtspunkt ist hierbei von Bedeutung, daß bei der Drittschadensliquidation durch die Einbeziehung des Dritten nur eine (zufällige) Schadensverlagerung eintritt, die dem Schädiger keine Mehrbelastung auferlegt. Beim Vertrag mit Schutzwirkung für Dritte kommt der Dritte dagegen als zusätzlicher Ersatzberechtigter mit einem eigenen Schaden in Betracht. Die Formel, daß bei der Drittschadensliquidation der Schaden zur Anspruchsgrundlage gezogen wird, während beim Vertrag mit Schutzwirkung für Dritte die Anspruchsgrundlage zum Schaden gezogen wird, ${ }^{72}$ veranschaulicht zwar, wer der jeweils Anspruchsberechtigte ist, gibt aber keine Kriterien dafür, wann das eine und wann das andere zur Anwendung kommt.

\section{Gründe für die Entwicklung im Schadensersatzrecht}

Fragt man sich, warum das Schadensersatzrecht eine solche Bedeutung erlangt hat und warum im Vergleich zu früher die Zahl der ungewöhnlichen Fälle, die den Gerichten zur Entscheidung unterbreitet wurden, zugenommen hat und Schadensersatz zumeist in erweitertem Umfang auch höchstrichterlich zugesprochen wurde, so ist dies sicher durch eine Fülle von Faktoren beeinflußt.

Gerichte können immer nur die Fälle entscheiden, die ihnen durch die Klage einer Partei unterbreitet werden. Deshalb liegt ein erster Faktor wohl im Wandel des gesellschaftlichen Bewußtseins, das einerseits wirtschaftlichen Faktoren größere Beachtung gewährt und andererseits für immer mehr Risiken Schutz und Ersatz durch andere sucht. So sind auch die Fälle, in denen ein Schadensersatz abgelehnt wird, weil der Geschädigte das Lebensrisiko selbst zu tragen hat, eher selten. ${ }^{73}$ Die Verwirklichung eines eigenen Lebensrisikos, das einen Schadensersatzanspruch ausschließt, wird etwa angenommen, wenn infolge des schädigenden Eingriffs, aber nicht durch diesen indiziert eine weitere Behandlung (z. B. eine Impfung ${ }^{74}$ ) vorgenommen wird oder ein weiterer Schaden entdeckt wird, der unabhängig

70 BGH NJW 2004, 3420.

71 § 328 Rn 117; ihm folgend Janoschek, in: Bamberger/Roth (Fn. 4), § 328 Rn. 54.

72 S. Brox/Walker (Fn. 8), § 33 Rn. 18 im Anschluß an Medicus, Bürgerliches Recht, Rn. 839.

73 Vgl. dazu den Überblick bei Palandt-Heinrichs (Fn. 3), Vorb. vor § 249 Rn. 88.

${ }^{74}$ BGHZ 25, 86; BGH NJW 1963, 1671. 
vom schädigenden Eingriff latent bereits vorhanden war (z. B. eine bereits vorhandene Krankheit $\left.{ }^{75}\right)$.

Eine weitere Ursache liegt aber auch im gewandelten dogmatischen Verständnis der Juristen, das sich unter dem Einfluß grundrechtlicher Wertungen stark verfeinert hat. Das gewandelte Verständnis findet vor allem in der Rechtswissenschaft seinen Ausdruck, aber auch in Urteilen werden am Fall neue Gedanken entwickelt. Von dort werden sie auch in den Lehrbüchern und Kommentaren aufgegriffen, verarbeitet und mit neuen Ideen angereichert. Diese Darstellungen flieBen sowohl in die Lehre als auch in die Praxis ein und bereiten so den Boden für neue Einschätzungen und Bewertungen, die aus der Anwaltschaft heraus den Weg zu den Gerichten finden. Auf diese Weise leisten gerade auch die Lehrbücher und Kommentare des Verlags einen wichtigen Beitrag zur Rechtsentwicklung.

\section{III. § 242 BGB als Grundlage für die Fortentwicklung des Schuldrechts}

Schon beim Einfluß der Grundrechte auf das Privatrecht war $§ 242$ BGB als Einfallstor für neue Wertungen genannt worden. § 242 BGB bot Rechtsprechung und Wissenschaft aber auch die rechtliche Grundlage, um anderen Bedürfnissen nach Weiterentwicklung des Rechts Rechnung zu tragen. Da § 242 BGB nach übereinstimmender Meinung nicht auf das Schuldrecht begrenzt ist, sondern im gesamten Rechtssystem seine Wirkung entfaltet, stützen sich eine Anzahl von Rechtsfiguren auf $\S 242$ BGB, die über das Schuldrecht hinaus Geltung erlangt haben. Dies gilt etwa für die verschiedenen Fallgruppen der unzulässigen Rechtsausübung. Diese waren zum Teil schon dem römischen Recht bekannt, haben aber in ihrer praktischen Relevanz eine weit darüber hinausgehende Bedeutung erlangt. Auch der Wegfall der Geschäftsgrundlage ist auf $§ 242$ BGB gestützt worden, selbst wenn er im Zusammenhang mit dem beiderseitigen Motivirrtum der Rechtsgeschäftslehre zuzurechnen war, seit der Schuldrechtsmodernisierung aber gleichwohl in das Allgemeine Schuldrecht ( $\$ 313$ BGB) eingefügt worden ist. Der $B G H$ hat auch die Inhaltskontrolle von Allgemeinen Geschäftsbedingungen auf $\S 242$ BGB gestützt, bevor sie unter Übernahme der wesentlichen Grundsätze gesetzlich geregelt wurden.

Dem Allgemeinen Schuldrecht zugerechnet werden kann aber die Figur des Einwendungsdurchgriffs, weil er die Relativität des Schuldverhältnisses durchbricht. Auch wenn der Einwendungsdurchgriff inzwischen in $§ \S 358,359$ BGB eine gesetzliche Regelung erfahren hat, so ist die Entwicklung dieses Rechtsinstituts aus $\S 242$ BGB doch von Interesse, weil sie für das Verständnis und die Auslegung der $\S \S 358,359$ BGB Anhaltspunkte liefern kann. Zudem ist die gesetzliche Regelung auf die Verbraucherverträge zwischen einem Unternehmer und einem Verbraucher beschränkt und es bleibt deshalb die Frage, ob der Einwendungsdurchgriff in anderen Vertragskonstellationen, etwa bei Verträgen zwischen Unternehmern oder zwischen Verbrauchern nicht nach wie vor über $\S 242 \mathrm{BGB} z u$ gewähren ist. Dies ist deswegen von Interesse, weil der vom $B G H$ entschiedene

75 BGH NJW 1968, 2287. 
Fall aus dem Jahre 1962 aus heutiger Sicht keinen Verbrauchervertrag betraf. Vielmehr ging es um den finanzierten Kauf von Mobiliar für eine Gaststätte, was der gewerblichen Tätigkeit zugerechnet werden muß.

In einer ersten Entscheidung aus dem Jahre $1956^{76}$ hatte sich der $B G H$ vor allem auf den Schutzzweck der Sachmängelgewährleistungsansprüche, der in Form eines Nachbesserungsrechts vereinbart war, gestützt und die Versagung des Einwendungsdurchgriffs gegenüber der finanzierenden Bank als Umgehung des Sachmängelrechts angesehen. ${ }^{77}$ Genauere Kriterien für die Verbindung der beiden Verträge hat der $B G H$ damals noch nicht herausgearbeitet. Erst später hat er in seinen Entscheidungen maßgeblich darauf abgestellt, daß die Erstreckung von Einwendungen aus einem Vertrag auf einen anderen Vertrag eine auf Dauer angelegte wirtschaftliche Verbindung der beiden Verträge, des finanzierten Geschäfts und des seiner Finanzierung dienenden Kreditvertrags, voraussetzt. ${ }^{78}$ Die Versagung des Einwendungsdurchgriffs in einem solchen Fall widerspreche dem in $§ 242$ BGB niedergelegten Prinzip von Treu und Glauben. Den Einwendungsdurchgriff hat der BGH aber in Verträgen mit Vollkaufleuten nicht gewähren wollen.

Später hat der $B G H$ seine Kriterien für die Verbindung des finanzierten mit dem finanzierenden Vertrag und den darauf gestützten Einwendungsdurchgriff präzisiert und verallgemeinert. Voraussetzung für die wirtschaftliche Verbindung der beiden Verträge sei das Vorliegen objektiver und subjektiver Verbindungselemente. Die objektiven Verbindungselemente stellen im wesentlichen auf das Zusammenwirken des finanzierenden Kreditinstituts mit dem Lieferanten oder sonstigen Leistungserbringer ab. Diese Voraussetzung enthält nunmehr $\S 358$ Abs. 3 BGB. Das subjektive Verbindungselement wird durch die Vorstellung des Leistungsempfängers geschaffen, die beiden Verträge bildeten eine rechtliche Einheit. ${ }^{79}$ Dies wird in $§ 358$ Abs. 3 BGB nicht mehr ausdrücklich erwähnt.

Die Entscheidungen sind nicht nur für die praktische Rechtsanwendung von Bedeutung. Sie berühren vielmehr und greifen in ganz grundsätzlicher Weise ein in die dogmatischen Grundlagen der Relativität des Schuldverhältnisses, die dadurch gekennzeichnet ist, daß Rechte und Pflichten nur in der Verbindung zwischen dem Gläubiger und dessen Schuldner bestehen, was gemäß der Trennungstheorie ein Übergreifen der Einwendungen aus dem einen Schuldverhältnis in ein anderes ausschließt. Trotz dieses grundsätzlichen Bruchs mit den allgemeinen Grundlagen des Schuldverhältnisses wird der Einwendungsdurchgriff in den Lehrbüchern des Allgemeinen Schuldrechts nicht angesprochen, sondern in das Besondere Schuldrecht verwiesen. ${ }^{80}$ Der Gesetzgeber des Schuldrechtsmodernisierungsgesetzes hat dies anders und richtig gesehen und die Regelung des Einwendungsdurchgriffs in das Allgemeine Schuldrecht eingestellt, dem Brox/Walker in seiner Darstellung folgt. ${ }^{81}$

\footnotetext{
76 S. BGHZ 22, 90.

77 BGHZ 22, 90, 100.

${ }^{78}$ BGHZ 37, 94, 101; s. zuvor schon BGHZ 22, 90, 100.

79 S. dazu BGH NJW 1980, 1155, 1157; 1983, 2250, 2251; BGH NJW-RR 1990, 1072.

80 So ausdrücklich Medicus (Fn. 48), Rn. 579.

81 Brox/Walker (Fn. 8), § 19 Rn. 42 ff., 47.
} 


\section{Entwicklungen im Recht der Forderungsabtretung}

Das Recht der Abtretung unterliegt einer verhältnismäßig konstanten Rechtsentwicklung. Die Auseinandersetzung der Rechtsprechung mit den Lehrbüchern und Kommentaren zum Allgemeinen Schuldrecht ist in diesem Bereich deshalb eher gering. Die Abtretung kann aufgrund ihrer Abstraktheit aber auch zu Sicherungszwecken eingesetzt werden kann. Die Sicherungsabtretung und der dazu gehörende Sicherungsvertrag, der eigentlich als besonderer Vertragstyp dem besonderen Schuldrecht zuzurechnen wäre, werden aber von den Lehrbüchern und Kommentaren bei der Abtretung im Allgemeinen Teil des Schuldrechts behandelt. Hinsichtlich der Abtretung als Verfügungsgeschäft ist vor allem die Erweiterung der Abtretbarkeit bedeutsam gewesen, indem hinsichtlich des Kreises der abzutretenden Forderungen in der Rechtsprechung auf die Bestimmtheit im Zeitpunkt des Abtretungsvertrags verzichtet wurde. Statt dessen ist von der Rechtsprechung die Bestimmbarkeit in dem Sinne als ausreichend angesehen worden, daß bei Abschluß des Abtretungsvertrags die Kriterien festgelegt sein müssen, aufgrund derer erst beim späteren Entstehen der Forderungen und der dann eintretenden Abtretungswirkungen bestimmbar ist, welche Forderungen von der Abtretung erfaßt werden. ${ }^{82}$

Aufgrund dieser Auflockerung wurde die vor allem zu Sicherungszwecken eingesetzte Vorausabtretung künftiger Forderungen und die Globalzession eines umfassenden Bündels von Forderungen wesentlich erleichtert. Diese Entwicklung hat sich ebenfalls in Auseinandersetzung mit der Wissenschaft vollzogen, wobei die Ausgangsentscheidung von 1952 noch das Lehrbuch von Enneccerus/Lehmann berücksichtigt. Lehrbücher und Kommentare des Verlags übernehmen aber diese Rechtsprechung. Umstritten ist noch, ob der Zedent der im voraus abgetretenen, aber noch nicht entstandenen Forderung seine Rechtsposition aus der Vorausabtretung seinerseits durch Abtretung auf andere übertragen kann. ${ }^{83}$ Die Frage dürfte $\mathrm{zu}$ bejahen sein, weil der Zedent schon eine Rechtsposition erlangt hat, über die er verfügen kann, zumindest liegt in der Vorausabtretung an den ersten Zedenten die Ermächtigung des Zessionars gemäß $§ 185$ BGB, daß dieser über die künftige Forderung verfügen darf.

Die Verfügung über die Forderung kann durch Vereinbarung zwischen Gläubiger und Schuldner ausgeschlossen werden. Davon wird im Geschäftsverkehr vielfach - zumeist in AGB - Gebrauch gemacht, weil der Schuldner seine Buchhaltung nicht umstellen will und vor allem bei Teilabtretungen sich nicht mehreren Gläubigern ausgesetzt sehen will. Die Rechtsprechung hat derartige Abtretungsverbote großzügig zugelassen. ${ }^{84}$ Dies hat den Gesetzgeber veranlaßt, mit der Vorschrift des $§ 354$ a HGB die Abtretung trotz des Abtretungsverbots bei beiderseitigen Handelsgeschäften für wirksam zu erklären.

Umstritten sind vor allem noch die Folgen eines Verstoßes gegen das Abtretungsverbot. Richtigerweise ist mit der Rechtsprechung von einer absoluten Ver-

\footnotetext{
82 BGHZ 7, 365; 26, 178

83 Bejahend Larenz (Fn. 22), § 34 III; einschränkend Medicus (Fn. 48), Rn. 713.

${ }^{84}$ Krit. Wolf/Horn/Lindacher, AGB-Gesetz, 4. Aufl. 1999, § 9 Rn. A 14.
} 
fügungsbeschränkung auszugehen, die eine gegen das Abtretungsverbot verstoBende Verfügung nicht nur relativ, sondern absolut unwirksam macht. ${ }^{85} \mathrm{Nicht}$ weiterfuhrend ist die Annahme, daß dadurch der Forderung die Verkehrsfähigkeit genommen werde. ${ }^{86}$ Davon zu unterscheiden ist die Frage, ob der Schuldner eine gegen das Abtretungsverbot verstoßende Abtretung nachträglich gemäß $§ 185$ Abs. 2 BGB genehmigen kann mit der Folge, daß die Abtretung rückwirkend wirksam wird ( $\$ 184$ Abs. 1 BGB). Entgegen der Rechtsprechung und einer verbreiteten Meinung ${ }^{87}$ ist dies $\mathrm{zu}$ bejahen $^{88}$. Die umständliche Konstruktion eines Vertrags über die Aufhebung des Abtretungsverbots ${ }^{89}$ erscheint entbehrlich. Gegen die rückwirkende Genehmigung könnte sprechen, daß dadurch eine zwischenzeitliche Verfügung des alten Gläubigers oder eine gegen ihn vorgenommene Pfändung unwirksam wird. Da ein gutgläubiger Erwerb von Forderungen ausgeschlossen ist, könnte der Schutz zwischenzeitlicher Erwerber ein Verbot der rückwirkenden Genehmigung rechtfertigen. Den Interessen zwischenzeitlicher Erwerber wird jedoch durch $\S 184$ Abs. 2 BGB Genüge getan, indem dort ausdrücklich angeordnet wird, daß zwischenzeitlich vorgenommene Verfügungen oder Maßnahmen der Zwangsvollstreckung ihre Wirksamkeit behalten. Die Lehrbuch- und Kommentarliteratur des Verlags bietet auf diese Weise die Vielfalt der Meinungen zuverlässig ab und gibt Anregungen zur wissenschaftlichen Auseinandersetzung.

\section{Schuldrechtsmodernisierung}

Wesentliche Änderungen im Allgemeinen Schuldrecht hat das im Jahre 2002 in Kraft getretene Schuldrechtsmodernisierungsgesetz gebracht. Eine erste Änderung betrifft die Einfügung von zuvor in Spezialgesetzen enthaltenen Vorschriften und die Umsetzung von EG-Richtlinien direkt in das BGB. Dazu gehören etwa die Vorschriften über die Inhaltskontrolle von Allgemeinen Geschäftsbedingungen (\$§ $305 \mathrm{ff}$. BGB), ebenso die Bestimmungen über den Widerruf von Haustürgeschäften und anderen Geschäften ( $\$ 312$ BGB) sowie die Vorschriften über den Fernabsatz und den elektronischen Geschäftsverkehr ( $\$ 312$ b ff. BGB). Zugleich ist durch die Regelung des Widerrufsrechts und seiner Rechtsfolgen in den $\S \S 355 \mathrm{ff}$. BGB und durch die Regelung des Einwendungsdurchgriffs in den $\S \S 358,359$ BGB die Funktion des Allgemeinen Schuldrechts gestärkt worden als der Ort, an dem die allgemeinen, für verschiedene Vertragsverhältnisse anwendbaren Vorschriften zu finden sind. Zusätzlich hat das BGB dadurch auch die Funktion des privatrechtlichen Verbraucherschutzes übernommen.

${ }^{85}$ BGHZ 56, 176; 102, 301; 108, 176; Larenz (Fn. 22), § 34 II 1; Palandt-Heinrichs (Fn. 3), § $399 \mathrm{Rn} 11$.

86 S. etwa $B G H Z 40,156,160 ; 108,172 ; 112,387$.

87 BGHZ 108, 176; Larenz (Fn. 22), § 34 II 1; Palandt-Heinrichs (Fn. 3), § 399 Rn. 11; a. A. noch $B G H$ NJW-RR 1991, 764.

88 Medicus (Fn. 48), Rn. 718; Brox/Walker (Fn. 8), § 34 Rn. 12; ohne Stellungnahme MünchKommBGB-Roth (Fn. 4), § 399 Rn. 37.

${ }^{89}$ S. etwa Larenz (Fn. 22), § 34 II 1; Palandt-Heinrichs (Fn. 3), § 399 Rn. 11. 
Eine weitere wesentliche Änderung betrifft das Recht der Leistungsstörungen. Hier ist das Gesetz so formuliert, daß auch die positive Vertragsverletzung mit geregelt ist. Ferner sind die Vorschriften über den gegenseitigen Vertrag so gefaßt worden, daß sich dort nicht mehr die Anspruchsgrundlagen für Schadensersatzansprüche, sondern nur noch die gesetzlichen Rücktrittsgründe befinden. Als wesentliche Änderung ist dabei zu vermerken, daß der Rücktritt im Gegensatz zu den bisherigen $\S \S 325,326$ BGB kein Verschulden und keine Ablehnungsandrohung wie in $\$ 326$ a. F. BGB mehr voraussetzt. Ausdrücklich vorgesehen ist statt dessen, daß Schadensersatzansprüche durch die Ausübung des Rücktrittsrechts nicht ausgeschlossen werden.

Geändert ist auch die Regelung über die anfängliche Unmöglichkeit in $§ 311 \mathrm{a}$ BGB. Anfängliche Unmöglichkeit führt jetzt nicht mehr zur Nichtigkeit des Vertrags wie in $\$ 306$ BGB a. F., sondern begründet einen Anspruch auf Schadensoder Aufwendungsersatz. Die Vorschrift über die Haftung ohne Verschulden hinsichtlich der Beschaffung von Gattungsschulden ( $\$ 279$ BGB a. F.) ist durch die Garantieübernahme in $\S 276$ Abs. 1 BGB ersetzt worden. In $\S 241$ a BGB findet sich eine Vorschrift über den Umgang mit unbestellten Leistungen. § 313 BGB regelt die Störung der Geschäftsgrundlage und § 314 BGB das Recht der fristlosen Kündigung aus wichtigem Grund. Schließlich ist noch die Aufnahme der culpa in contrahendo in $\S 311$ Abs. 2 und 3 BGB zu erwähnen, wobei unterschiedliche Meinungen darüber bestehen, ob § 311 Abs. 3 BGB auch den Vertrag mit Schutzwirkung für Dritte enthält, was vom Gesetzgeber aber wohl nicht beabsichtigt war.

Die Lehrbücher und Kommentare des Verlages haben die neuen Vorschriften eingearbeitet und bieten so eine wertvolle Hilfe im Studium und in der Praxis beim Verständnis der neuen Vorschriften. Das Lehrbuch von Larenz zum Allgemeinen Schuldrecht liegt allerdings noch in 14. Auflage 1987 vor.

Angesichts der kurzen Zeit seit dem Inkrafttreten der neuen Vorschriften liegen $\mathrm{zu}$ wichtigen Fragen noch keine höchstrichterlichen Entscheidungen vor. Eine Entscheidung zu $\S 312 \mathrm{~d}$ BGB betreffend das Widerrufsrecht bei Internet-Auktionen kommt zwar ganz ohne Auseinandersetzung mit der Literatur aus. ${ }^{90}$ In einer weiteren BGH-Entscheidung zu $§ 312$ b BGB, in der das Postident-Verfahren dem Fernabsatz zugerechnet wird, wird die Kommentar-Literatur des Verlages (Münchener Kommentar, Bamberger/Roth, Palandt) aber mehrfach verarbeitet. ${ }^{91}$ Sie dokumentiert auf diese Weise nach wie vor ihren Einfluß auf die höchstrichterlichen Entscheidungen gerade in ersten Entscheidungen zu neuen Vorschriften.

Die Aufnahme des AGB-Rechts ins BGB macht deutlich, daß die Inhaltskontrolle von Verträgen ein wesentlicher Bestandteil des Zivilrechts ist. Die etwas unglückliche Einfügung in das Allgemeine Schuldrecht statt in den Allgemeinen Teil des BGB darf nicht dazu verleiten, die Inhaltskontrolle auf Schuldverträge zu begrenzen. Sie ist auch auf Regelungsmaterien des Sachenrechts und des Allgemeinen Teils des BGB anzuwenden. Dies wird in den Lehrbüchern zum Schuldrecht

90 BGH NJW 2005, 53 m. Anm. Schimmel/Buhlmann, LMK 2005, 2.

91 BGH NJW 2004, 3699 m. Anm. Schulte-Nölke, LMK 2005, 20. 
zu Recht betont. ${ }^{92}$ Auf eine ausführliche Behandlung des AGB-Rechts in den Schuldrechtslehrbüchern wird weitgehend verzichtet. ${ }^{93}$

Inhaltlich bringen die ins BGB eingefügten Vorschriften der $\S \S 305 \mathrm{ff}$. BGB keine wesentlichen Änderungen gegenüber dem früheren AGB-Gesetz. Besonders hingewiesen sei jedoch darauf, daß nunmehr die Anwendung der Vorschriften über die Inhaltskontrolle von AGB in $\S 310$ Abs. 4 Satz 2 BGB auch für Arbeitsverträge vorgesehen ist. Ausdrücklich aufgenommen wurde zudem die Berücksichtigung des Transparenzgebots bei der Inhaltskontrolle ( $\$ 307$ Abs. 1 Satz 2 BGB). Im übrigen wurden die Klauselverbote der $\$ \S 308$ und 309 BGB den geänderten Vorschriften des Schuldrechts angepaßt. Eine bemerkenswerte Folge der neuen Vorschriften zum Verbraucherschutzrecht im BGB ist, daß sich der Gesetzgeber dabei wie schon im Mietrecht vielfach der halbzwingenden Normen bedient. Deren Kennzeichen ist, daß sie nur zugunsten des Verbrauchers abbedungen werden können, jede Abweichung zum Nachteil des Verbrauchers aber schon von Gesetzes wegen ausgeschlossen ist. Dies schränkt jeglichen Gestaltungsspielraum in AGB ein und macht die richterliche Inhaltskontrolle von AGB überflüssig. Diese Einschränkung der Gestaltungsfreiheit, die trotz der Inhaltskontrolle von AGB immer noch in größerem Umfang als bei halbzwingenden Normen besteht, wird zu wenig beachtet.

\section{Ein Blick in die Zukunft}

Ein historischer Rückblick in die Vergangenheit reizt natürlich auch dazu, einen Blick in die Zukunft zu werfen. Wie wird sich der Markt für Publikationen entwickeln und welche Stellung wird der Verlag dabei einnehmen? Ein solcher Blick in die Zukunft ist ein Wagnis für den, der ihn unternimmt, weil Prognosen leicht in die Irre gehen können. Prognosen sind dennoch interessant, weil sie Orientierungspunkte für gegenwärtiges, zukunftsgerichtetes Handeln, insbesondere für Investitionsentscheiden bieten können. Eine vorausschauende Zukunftsbetrachtung kann aber auch für künftige Leser von Interesse sein, nicht nur weil sie aus späterer Sicht das Eintreffen oder Nichteintreffen der Prognosen ähnlich wie bei einem Horoskop zu beurteilen vermögen, sondern auch weil ihnen aufgrund der Prognosen ein Einblick in das Denken der Zeit mit ihren Erwartungen und vielleicht auch Sorgen ermöglicht wird. Der Blick in die Zukunft soll sich auf drei Bereiche richten.

\section{Zukunft des Allgemeinen Schuldrechts}

Zunächst soll die mögliche Entwicklung des Allgemeinen Schuldrechts betrachtet werden. Dieses Rechtsgebiet wird sich im Grundsatz sicher seine Existenz bewahren, so wie es schon seit dem römischen Recht Bestand gehabt hat. Zwar werden

92 S. Lorenz/Riehm, Lehrbuch zum neuen Schuldrecht, 2002, Rn. 91; Brox/Walker (Fn. 8), $\S 4$ Rn. 28.

${ }^{93}$ S. auch Medicus (Fn. 48), Rn. 90 f. 
sich wie in den letzten einhundert Jahren immer wieder Entwicklungen ergeben, die dem technischen und wirtschaftlichen Wandel Rechnung tragen. Jedoch werden sich die rechtlichen Grundlagen des Schuldverhältnisses und seiner Erfüllung sowie die Folgen von Pflichtverletzungen und die Fungibilität von Forderungsrechten nicht wesentlich ändern. Der gegenwärtig erkennbare Trend zur Arbeitsteilung und Spezialisierung wird aber wohl die Relativität und isolationistische Betrachtung des einzelnen Schuldverhältnisses aufweichen und neue Regeln bezüglich des funktionalen Zusammenwirkens von Verträgen entstehen lassen, wie sie ansatzweise beim Einwendungsdurchgriff schon gegeben sind. Das Phänomen der Netz- und Kettenverträge bietet hierfür schon Anschauungsmaterial und Herausforderungen. ${ }^{94}$

Weitere Veränderungen werden sich wohl auf dem Gebiet der europäischen Rechtsharmonisierung ergeben, ja vielleicht wird das Allgemeine Schuldrecht sogar an einer durch den Handels- und Wirtschaftsverkehr veranlaßten globalen Vereinheitlichung teilnehmen. Auf der europäischen Ebene dürften die Arbeiten an einem europäischen Zivilgesetzbuch weitere Fortschritte machen. Dabei sollte eine Vereinheitlichung des Allgemeinen Schuldrechts keine allzu großen Schwierigkeiten bereiten, da die Probleme und Lösungen in den verschiedenen Rechtsordnungen vergleichbar sind und dogmatische Besonderheiten bei der praktischen Rechtsanwendung vielfach an Gewicht verlieren. Der Verlag C. H. Beck ist mit seinen Lehrbüchern und Kommentaren im Bereich des europäischen Privatrechts und der europäischen Rechtsharmonisierung noch nicht stark vertreten. Er hat aber im Nomos Verlag in diesem Bereich bereits eine Stütze, die sowohl ausgebaut werden kann als auch mit ihrer Erfahrung eine etwaige Umstellung beim Beck Verlag unterstützen kann

\section{Buchdruck und elektronische Medien}

Elektronische Medien und Datenbanken werden weiter auf dem Vormarsch sein. Der Zugriff auf Datenbanken über Online-Recherche und der Zugriff auf Datenträger mit gespeicherten Daten wird sicher den Zugriff auf Informationen in vielfacher Hinsicht erleichtern und beschleunigen. Eine spannende Frage ist, ob die neuen Medien das Buch und den Buchdruck nach mehr als 500 Jahren verdrängen und ersetzen werden. Für das Buch sprechen bisher immer noch einige Aspekte.

Man kann das Buch ohne technische Komplikationen überall hin mitnehmen. Die mit der elektronischen Datenverarbeitung verbundenen Probleme werden sich aber lösen lassen, so daß das elektronische Abfragen in gleicher Weise überall möglich sein wird wie beim Buch. Ein weiterer Vorzug des Buches ist seine Beständigkeit, die zugleich Grundlage seiner Zitierfähigkeit ist. Einmal gedruckt, ist das Buch immer wieder greifbar. Die gedruckten Daten gehen, abgesehen von der Vernichtung, nicht verloren und wenn ein Buch zerstört wird, gibt es immer noch mehrere andere.

94 S. dazu etwa Teubner, Netzwerk als Vertragsverbund, 2004; Rohe, Netzverträge, 1998; Larenz/Wolf, Allgemeiner Teil des bürgerlichen Rechts, 9. Aufl. 2004, § 23 Rn. $127 \mathrm{ff}$.

Manfred Wolf 
Die Beständigkeit erscheint aus heutiger Sicht bei den elektronischen Daten nicht in gleicher Weise gewährleistet. Es ist unklar, wer die elektronisch gespeicherten Daten sichert. Es müßte vergleichbar den Bibliotheken eine verantwortliche Stelle für die Sammlung und Erhaltung der elektronischen Daten geschaffen werden, damit auf sie ständig zugegriffen werden kann. Dies würde auch die Zitierfähigkeit ermöglichen. Es müßte dann noch die Manipulationsmöglichkeit durch den Zugriff von außen ausgeschlossen werden. Es spricht viel dafür, daß diese Fragen erfolgreich gelöst werden können. Ob das Buch dann endgültig verschwinden wird, ist damit wohl weniger eine Frage der technischen Möglichkeiten als vielmehr eine Frage der emotionalen Bindung und der Nostalgie.

\section{Zukunft des Verlages}

Die Zahl der Juristen hat wohl einen Höhepunkt erreicht, der trotz zunehmender Verrechtlichung und Bürokratisierung bei stagnierenden oder gar rückgängigen Bevölkerungszahlen nicht mehr ohne weiteres anwachsen wird. Der Verlag hat sich aber in der Vergangenheit eine starke Stellung erobert, die ihn in die Lage versetzen wird, auch die künftigen Herausforderungen zu bestehen. Selbst wenn das Allgemeine Schuldrecht auf der europäischen Ebene vereinheitlicht wird, muß den nationalen Bedürfnissen angesichts der Unterschiedlichkeit der Sprachen mit selbständigen Publikationen in der jeweiligen Sprache immer noch Rechnung getragen werden. Daneben wird es im Interesse der einheitlichen Verständigung und Rechtsanwendung sicher eine einheitliche Sprache gerade auch für Juristen geben. Diese Sprache wird aller Voraussicht nach auch im Rechtsbereich auf der Basis des Englischen ausgebildet werden, wobei manche dogmatischen Begriffe, die im englischen Recht nicht geläufig sind (z. B. Willenserklärung, Rechtsgeschäft, Gestaltungsrecht u.a.), aus anderen Sprachen übernommen oder neu gebildet werden.

Auf die gemeinschaftliche einheitliche Sprache werden sich die Autoren und mit ihnen der Verlag einstellen müssen. Für den Verlag stellt sich damit zugleich die Frage, wie er sich in dem neuen, von Publikationen in englischer Sprache geprägten größeren Markt behaupten kann. Mit den neuen elektronischen Medien, auf die sich der Verlag bereits eingestellt hat, dürfte die Verbreitung keine Schwierigkeiten bereiten. Für die Frage der Marktstärke dürfte vor allem wesentlich sein, ob der Verlag Autoren gewinnt, die im europäischen Raum Anerkennung finden oder schon gefunden haben. Dies dürfte ausgehend von der heutigen Marktstärke und der verbreiteten Anerkennung und Wertschätzung, die die Publikationen des Verlages in der Rechtsprechung, in der Anwaltschaft, in der Wissenschaft und in der Ausbildung genießen, eine lösbare Aufgabe sein. Der Erfolg auf diesem Weg ist dem Verlag zu wünschen. 